\title{
OPTIMIZING MOTION PLANNING FOR HYPER DYNAMIC MANIPULATOR
}

\author{
Souhila Aboura - Abdelhafid Omari \\ Kadda Zemalache Meguenni
}

\begin{abstract}
This paper investigates the optimal motion planning for an hyper dynamic manipulator. As case study, we consider a golf swing robot which is consisting with two actuated joint and a mechanical stoppers. Genetic Algorithm (GA) technique is proposed to solve the optimal golf swing motion which is generated by Fourier series approximation. The objective function for GA approach is to minimizing the intermediate and final state, minimizing the robot's energy consummation and maximizing the robot's speed. Obtained simulation results show the effectiveness of the proposed scheme.
\end{abstract}

K e y w or d s: robot motion planning, genetic algorithm, hyper dynamic manipulation, constrained optimization

\section{INTRODUCTION}

Recently, many investigations have been devoted in order to reproduce human body and to improve the capability of dynamic manipulator, especially to realize a high speed motion. Consequently, a new category of light weight and hyper dynamic manipulator is developed. This kind of robots, characterized by their high motion specification and smart structure, has become an interesting and challenging topic [1].

As case study, we use a golf swing robot, consisting with two actuated joint and a mechanical stoppers, which has been developed by Ming et al [2]. The motion of this robot is considered as an extension of hyper dynamic manipulation.

This robot interests many researchers. In [3] Suzuki and Inooka investigate a similar golf swing robot that could adjust its motion to both a specified value of swing velocity and specific characteristics of individual golf clubs. In those studies, wrist joint was considered as a passive joint containing only a brake mechanism that emulates the wrist cocking action of golfer, and it was assumed that the swing motion is executed by using dynamic interference forces. Suzuki and Inooka study also optimal feedforward torque plans utilizing shaft elasticity where the gravity is ignored, and they have shown that the brake mechanism and the flexibility of a golf club take important roles in the golf swing [4]. Hoshino and his team [5] discussed the vibration control problem of their golf swing robot and proposed an optimal control scheme using a state observer that considerer disturbance to suppress the vibration.

Most research works given above adopted, in motion control, predefined torque for each joints. However, In this case, the dynamic of robot is neglected. Therefore, the conventional methods, developed for motion generation, are not suitable for this kind of problem because they simplify golf swing planning and they lead to low robot dynamic performance. For these reasons, other researchers are centralized on how to determine the whole torque necessary to reflect the golfer arm's movement. Hence, that allowed realizing hyper dynamic manipulation with smart and versatile robot using unique mechanism design and control methods [1], [2], [6], [7], [8].

Comparing to conventional manipulators, the model and the mechanism of golf swing robot are based on the human ingenious structure, in which the actuators near body are more powerful and heavier than those near the end of arm. That is, the power/torque can be transferred from the body to the end of arm during hyper dynamic manipulation [2]. This torque is called dynamic coupled driving.

To validate this inspiration, the golf swing robot, considered in this study, consists of two joints, shoulder joint with powerful direct drive motor and wrist joint with a small direct drive motor [2]. The second joint is limited by mechanical stoppers in order to reproduce the human's wrist function. To realize high speed swing motions, it is necessary to use dynamically coupled driving which is defined as a transfer of power/ torque from the shoulder joint to the wrist joint.

In the present paper, we are most interesting in an optimal golf swing motion. This movement is constrained by actuator type, the strong nonlinearity due to the dynamically coupled driving torque, joint stops and the multiboundary condition of the motion specifications which must be taken into account. Hence we propose Genetic Algorithm (GA) to optimize the active torque input generated by Fourier series approximation.

The remainder of this paper is organized as follow. The robot's mechanism and model are introduced in Section 2. A new motion generation method to realize hyper dynamic manipulation based on constrained optimization using genetic algorithm with Fourier series approximation

* University of Sciences and Technology MB, Faculty of Electrical Engineering, Automatic Control Department, LDEE Laboratory, BP 1505 Oran El M’Naouer, Algeria, asouha79@yahoo.fr, o_abdelhafid@hotmail.com, zemalache@hotmail.com 

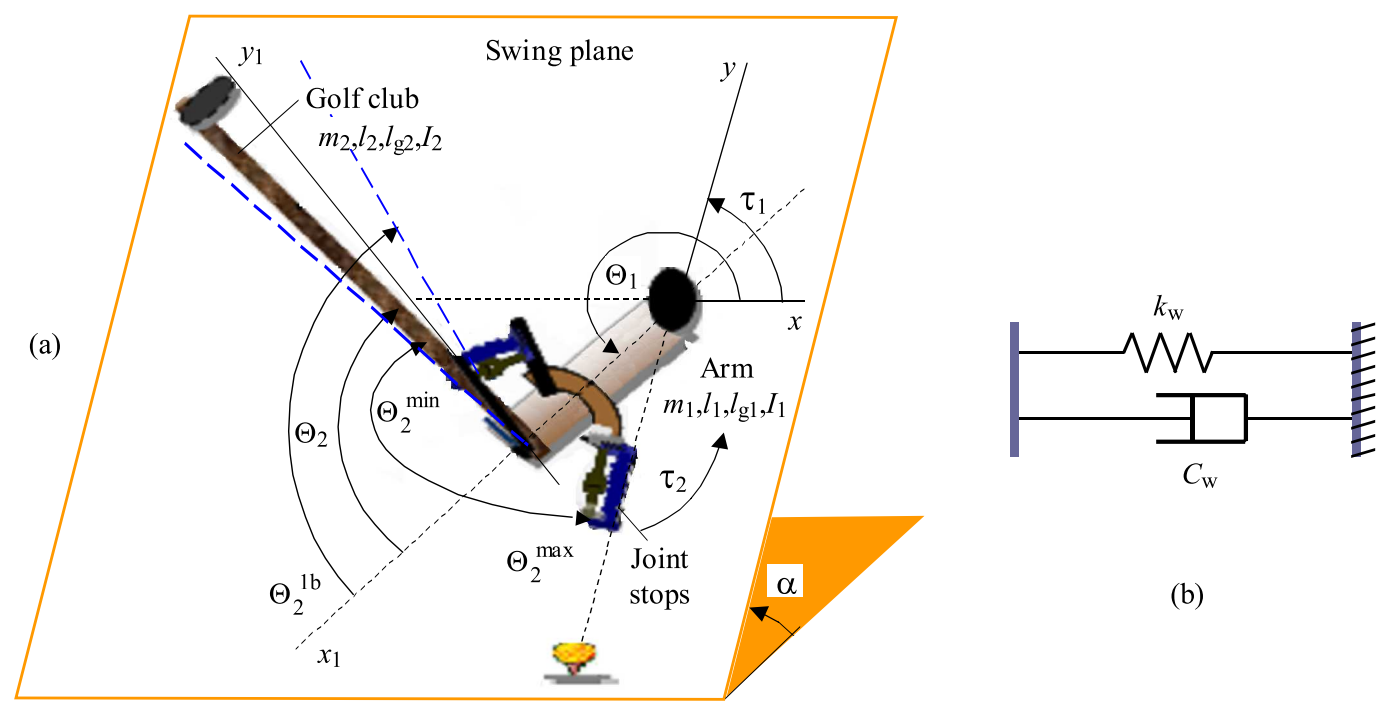

(b)

Fig. 1. Model of the Golf swing robot with joint stops

Table 1. Parameters of the robot

\begin{tabular}{lcc}
\hline Parameters & Symbol & Value \\
\hline Masse of arm $(\mathrm{kg})$ & $m_{1}$ & 4.5 \\
Moment of inertia of arm $\left(\mathrm{kgm}^{2}\right)$ & $I_{1}$ & 1.27 \\
Length of arm $(\mathrm{m})$ & $l_{1}$ & 0.4 \\
Location of centroid of arm $(\mathrm{m})$ & $l_{g 1}$ & 0.1333 \\
Masse of club $(\mathrm{kg})$ & $m_{2}$ & 1.24 \\
Moment of inertia of club $\left(\mathrm{kgm}^{2}\right)$ & $I_{2}$ & 0.00033 \\
Length of club $(\mathrm{m})$ & $l_{2}$ & 0.95 \\
Location of centroid of club $(\mathrm{m})$ & $l_{g 2}$ & 0.3167 \\
\hline
\end{tabular}

is described in Section 3. Simulation results of optimal motion generation are shown in Section 4. Finally, Section 5 concludes the results.

\section{MECHANISM AND MODEL OF ROBOT}

\subsection{Mechanism of Robot}

We consider a golf swing robot, consisting of two joints and joint stops, which is developed by Ming and his team [10]. This robot has an ingenious structure like human arm. So, the first joint (shoulder) is driven by a highpower direct drive motor, which is suitable for hyper dynamic manipulation, and the second joint (wrist) is driving by a light and low-power direct drive motor. Figure 1(a) shows the robot's prototype, and Tab. 1 gives its link parameters.

The rotation range of each joint $i,(i=1,2)$, is limited and it is given by (1).

$$
\theta_{i}^{\min } \leq \theta_{i} \leq \theta_{i}^{\max }, \quad(i=1,2)
$$

Where: $\theta_{i}(i=1,2)$ is the generalized coordinate of joints $i ; \theta_{i}^{\min }, \theta_{i}^{\max },(i=1,2)$ are the free rotation range of joint $i$ (minimum and maximum).
To reproduce efficiently the rotation range of golfer's wrist, the second joint, is limited by mechanical stoppers, Fig. 1(b). These stoppers are consisting of spring-damper arrangement which has an elastic characteristic [9]. Consequently, the joint stop's working rang is 20 degrees. This constraint is written as

$$
\begin{gathered}
\theta_{2}^{l b} \leq \theta_{2} \leq \theta_{2}^{\min } \\
\theta_{2}^{\max } \leq \theta_{2} \leq \theta_{2}^{u b} \\
\theta_{2}^{l b}(\mathrm{deg})=\theta_{2}^{\min }(\mathrm{deg})+20(\mathrm{deg}) \\
\theta_{2}^{u b}(\mathrm{deg})=\theta_{2}^{\max }(\mathrm{deg})-20(\mathrm{deg})
\end{gathered}
$$

$\theta_{2}^{l b}$ is the maximum of rotation range of the joint stop in clockwise direction. $\theta_{2}^{u b}$ is the maximum of rotation range of the joint stop in anticlockwise direction.

The active torque limits of actuators, given by (4), are important constraints for the realization of the ingenious structure in a hyper dynamic manipulation.

$$
\forall t \in\left[t_{0}, t_{1}\right], \tau_{i}^{\min } \leq \tau_{i}(t) \leq \tau_{i}^{\max },(i=1,2) .
$$

Where $\tau_{i}(t),(i=1,2)$ is the active torque of joint $i$; $\tau_{i}^{\max }, \tau_{i}^{\min },(i=1,2)$ are the maximum and the minimum active torque of joint $i$ respectively; $t_{0}, t_{f}$ are the time of initial and final position respectively.

\subsection{Golf Swing Motion}

The whole golf swing motion can be divided into three primary phases [9], Fig. 2.

Backswing Phase (from initial position to top position) In the beginning of this phase, both the club and the arm are vertically downward. Then the club is taken back from the initial position to its top position.

Downswing phase (from top position to impact position) This phase reflects the hyper dynamic manipulation. The club is swung down from the top position to the impact position to hit a golf ball. 

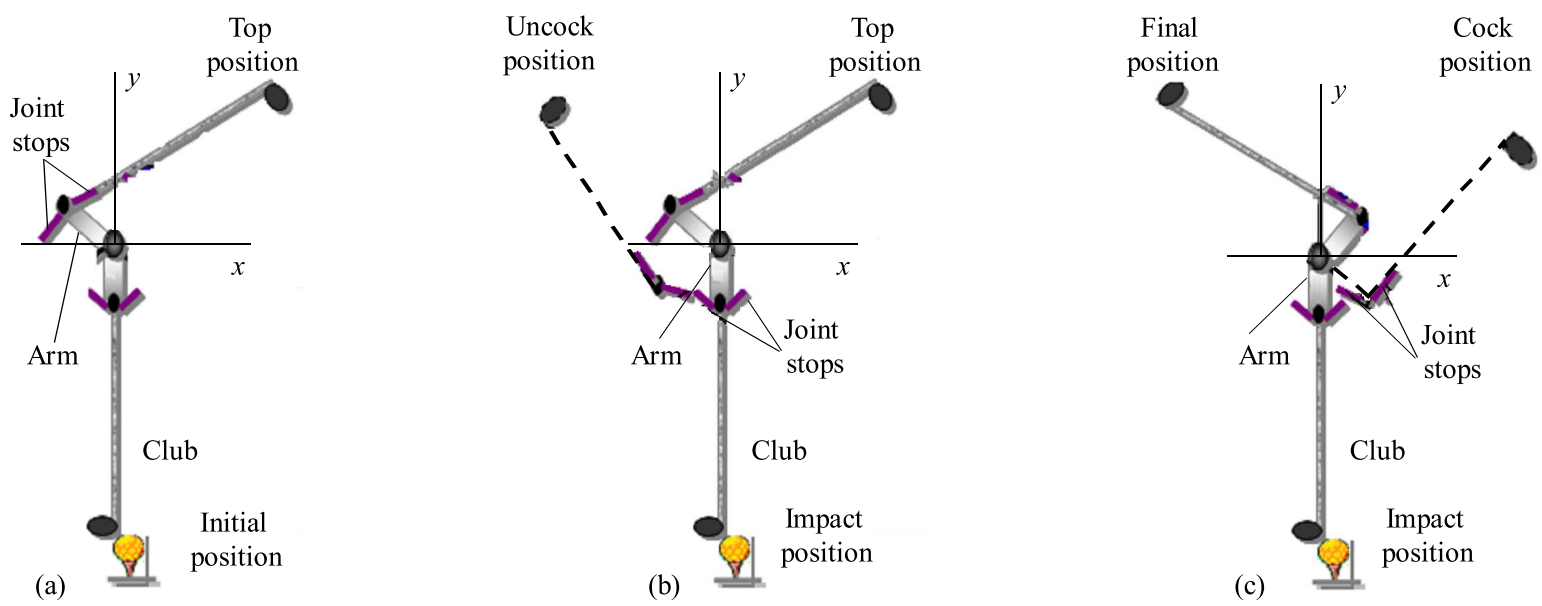

Fig. 2. Mechanism of Golf Swing Robot. (a) Backswing Phase, (b) Downswing phase, (c) Follow through phase

Follow through phase (from impact position to final position) After impacting the golf ball, the robot is slowed down and finally stopped.

\subsection{Model of the Robot}

A mathematical model of robot is

$$
\Gamma_{i}=M(\theta) \ddot{\theta}+N(\theta, \dot{\theta}) \dot{\theta}+G(\theta),(i=1,2) .
$$

Where $\Gamma_{1,2}=\left[\begin{array}{ll}\tau_{1} & \tau_{2}\end{array}\right]^{\top}$ is driving torque vector including active and passive torque. $\theta_{i}, \dot{\theta}_{i}, \ddot{\theta}_{i}$ are angle, angular velocity and acceleration of joint $i ; M(\theta)$ is the inertia matrix, $N(\theta, \dot{\theta})$ is the centrifuge and coriolis matrix and $G(\theta)$ is the gravity vector.

Mathematical model corresponding to the passive torque generated by joint stops is

$$
\begin{aligned}
& \tau_{2 \text { passif }}^{\min }=k_{w}\left(\theta_{2}-\theta_{2}^{\min }\right)-c_{w} \dot{\theta}_{2}, \\
& \tau_{2 \text { passif }}^{\max }=k_{w}\left(\theta_{2}-\theta_{2}^{\max }\right)-c_{w} \dot{\theta}_{2} .
\end{aligned}
$$

Where $k_{w}$ is stiffness scalar and $c_{w}$ is damping scalar; $\tau_{2 \text { passif }}^{\min }, \tau_{2 \text { passif }}^{\max }$ are the minimum and the maximum passive torque of joint 2 .

The torque of joint 2 is shown by

$$
\tau_{2}(t)= \begin{cases}\tau_{2 a c t i f}+\tau_{2 p a s s i f}^{\min }, & \theta_{2} \in\left[\theta_{2}^{l b}, \theta_{2}^{\min }\right), \\ \tau_{2 a c t i f}, & \theta_{2} \in\left[\theta_{2}^{\min }, \theta_{2}^{\max }\right], \\ \tau_{2 \text { actif }}+\tau_{2 \text { passif }}^{\max }, & \theta_{2} \in\left(\theta_{2}^{\max }, \theta_{2}^{u b}\right] .\end{cases}
$$

\section{OPTIMAL MOTION PLANNING}

\subsection{Problem Formulation}

The conventional motion planning, based on the robot's kinematics only, is carried out by decomposing the motion specifications of the end-effector and generating the joint trajectory according to a fixed motion profile. However, these methods are not pertinent in our case because the robot's active torque must be satisfied besides other motion constraints. We propose to use a new form of Fourier series to approximate the input active torques of joint 1 and joint 2 from the initial position to the final one

$$
\begin{aligned}
\tau_{1}(t) & =\sum_{i=1} n e a_{i} \sum_{n=0}^{n e / 2} b_{n} \cos \left(2 \pi f_{n}\left(t-d c_{i}\right)\right), \\
\tau_{2 a c t i f} & =\sum_{i=1} n e a_{i}^{\prime} \sum_{n=0}^{n e / 2} b_{n} \cos \left(2 \pi f_{n}\left(t-d c_{i}^{\prime}\right)\right) .
\end{aligned}
$$

Where $f_{n}=n f_{e} / n e, b_{1}, \ldots, b_{n e / 2}=2 / n e$ and $b_{0}=$ $1 / n e$. ne is number of samples corresponding to whole robot motion $\left[\begin{array}{ll}0 & t_{f}\end{array}\right] ; f_{e}$ is the sampling frequency; $\left(d c_{i}, d c_{i}^{\prime}\right)$ are times corresponding to each sample $(i=$ $1, n e) ;\left\{a_{i}, a_{i}^{\prime}\right\}$ are respectively the values of $\tau_{1}(t)$ and $\tau_{\text {2active }}(t)$ corresponding to each samples $(i=1, n e)$.

Optimal motion planning problem is transformed into a problem of obtaining the Fourier series coefficients, $\left\{a_{i}, a_{i}^{\prime}\right\}$, corresponding to active torques, with satisfying some constraints, boundary conditions and minimizing the chosen cost function. Torque $\tau_{2}(t)$ is obtaining, following (7). Then, the optimal motion of joint 1 and joint 2 can be derived by solving direct dynamic of robot.

\subsubsection{Boundary condition}

The boundary conditions are initial, impact and final configurations. For $(i=1,2)$

$$
\begin{array}{ll}
\theta_{i 0}, \dot{\theta}_{i 0}, \ddot{\theta}_{i 0}, & \text { initial configuration, } \\
\theta_{i m}, \begin{array}{l}
\left(v_{2 x m}=v_{2 x}\right), \\
\left(v_{2 y m}=v_{2 y}\right),
\end{array} & \text { impact configuration, } \\
\theta_{i f}, \dot{\theta}_{i f}, \ddot{\theta}_{i f}, & \text { final configuration. }
\end{array}
$$

Where $t_{m}$ is the impact time; $v_{2 x}$ and $v_{2 y}$ are the translational impact speed in $x$ and $y$ direction respectively; $v_{2 x m}$ and $\theta_{m}$ are the target impact speed, and the target impact position. 
Table 2. Boundary conditions

\begin{tabular}{cccc}
\hline & $\begin{array}{c}\text { Initial } \\
\text { configuration }\end{array}$ & $\begin{array}{c}\text { Impact } \\
\text { configuration }\end{array}$ & $\begin{array}{c}\text { Final } \\
\text { configuration }\end{array}$ \\
\hline$\theta(\mathrm{rad})$ & $\theta_{10}=3 \pi / 2$ & $\theta_{1 m}=3 \pi / 2$ & $4 \pi / 9 \leq \theta_{1 f} \leq 8 \pi / 3$ \\
& $\theta_{20}=2 \pi$ & $\theta_{2 m}=2 \pi$ & $4 \pi / 3 \leq \theta_{2 f} \leq 8 \pi / 3$ \\
$\dot{\theta}(\mathrm{rad} / \mathrm{s})$ & $\dot{\theta}_{10}=0$ & $v_{2 x m} \simeq 15, \ldots$, & $\dot{\theta}_{1 f}=0$ \\
& $\dot{\theta}_{20}=0$ & $40(\mathrm{~m} / \mathrm{s})$ & $\dot{\theta}_{2 f}=0$ \\
$\ddot{\theta}\left(\mathrm{rad} / \mathrm{s}^{2}\right)$ & $\ddot{\theta}_{10}=0$ & & $\ddot{\theta}_{1 f}=0$ \\
& $\ddot{\theta}_{20}=0$ & & $\ddot{\theta}_{2 f}=0$ \\
\hline
\end{tabular}

\subsubsection{Constraints}

To reproduce the professional golfer's motion, the robot must satisfy some constrains like those given by, $(1),(2),(3),(5),(6)$ in addition to the hard constraint on the active torques given by (4), which become

$$
\forall t \in\left[t_{0}, t_{f}\right],\left\{\begin{array}{l}
\tau_{1}^{\min } \leq a_{i} \leq \tau_{1}^{\max }, \\
\tau_{2 a c t i v e}^{\min } \leq a_{i}^{\prime} \leq \tau_{2 \text { active }}^{\max }
\end{array} \quad(i=1, n e)\right.
$$

\subsubsection{Cost Function}

To satisfy the robot's motion specifications, we suggest to minimize the impact and final error and to minimize the total consumed work or to maximize the impact speed. For $(i=1,2)$ we have

Impact error: $\Theta_{m}=$

$$
\left[\theta_{i m}-\theta_{i}\left(t_{m}\right) ; v_{2 x}\left(t_{m}\right)-v_{2 x m} ; v_{2 y}\left(t_{m}\right)-v_{2 y m}\right] .
$$

Final error: $\quad \Theta_{f}=\left[\dot{\theta}_{i f}-\dot{\theta}_{i}\left(t_{f}\right) ; \ddot{\theta}_{i f}-\ddot{\theta}_{i}\left(t_{f}\right)\right]$.

Total consumed work: $J_{c 1}=$

$$
\int_{0}^{t_{f}}\left|\tau_{1}(t) \dot{\theta}_{1}(t)\right| \mathrm{d} t+\int_{0}^{t_{f}}\left|\tau_{2 a c t i f}(t) \dot{\theta}_{2}(t)\right| \mathrm{d} t .
$$

Impact speed: $\quad J_{c 2}=\left|1 / v_{x 2}\left(t_{m}\right)\right|$.

First cost function (error, total work):

$$
J_{1}=\Theta_{m}^{\top} w_{1} \Theta_{m}+\Theta_{f}^{\top} w_{2} \Theta_{f}+w_{3} J_{c 1}
$$

Second cost function (error, impact speed):

$$
J_{2}=\Theta_{m}^{\top} w_{1} \Theta_{m}+\Theta_{f}^{\top} w_{2} \Theta_{f}+w_{4} J_{c 2} .
$$

Where $w_{1}, w_{2}, w_{3}$ and $w_{4}$ are the weighting matrix. Finally, the formulation problem of an optimal motion planning for the robot is given by

$$
\min _{\left(\left\{a_{i}, a_{i}^{\prime}\right\}\right)_{i=0, n e}} J_{1,2}\left(a_{i}, a_{i}^{\prime}\right)
$$

subject to $(1),(2),(3),(5),(6),(7),(10)$ and (11).

\subsubsection{Optimization Method}

To solve the optimal golf swing given by (18), Genetic algorithms method is applied. Compared to traditional search and optimization procedures, the GA is robust and global technique. GA does not require derivative information or a formal initial estimate of the solution region and because of the stochastic nature of the search mechanism, the GA is capable of searching the entire solution space with more likelihood of finding the global optimum [10]. GA planning scheme render an optimizing motion generation of golf swing robot having, in addition to minimum impact and final error, minimum total work or maximum speed impact.

\section{SIMULATION RESULTS}

To study the characteristics of the hyper dynamic manipulation, first, we choose the impact and the final time $\left(t_{f}=1.4 \mathrm{~s}\right)$ and the various speeds $\left(v_{2 x m} \simeq\right.$ $15, \ldots, 40(\mathrm{~m} / \mathrm{s}))$ corresponding to impact position. Then we discretize the time interval of the whole robot motion as to use 8 samples $(n e=8)$. We fixed also the parameters: $f_{e}=5 \mathrm{~Hz}$, and

$$
d c_{i}=d c_{i}^{\prime}=\left\{\begin{array}{llllllll}
0 & 0.2 & 0.4 & 0.6 & 0.8 & 1 & 1.2 & 1.4
\end{array}\right\} \mathrm{s} .
$$

The main parameters for the simulation and the boundary conditions are given in Table 2 and the following equations

$$
\begin{aligned}
\left|\tau_{1}\right| & \leq 110(N . m) ;\left|\tau_{2}\right| \leq 11(N . m) \\
\theta_{1}^{\min } & =80, \theta_{1}^{\max }=460, \theta_{2}^{\min }=260(\mathrm{deg}), \\
\theta_{2}^{\max } & =460, \theta_{2}^{l b}=240, \theta_{2}^{u b}=480(\mathrm{deg}) .
\end{aligned}
$$

The optimal golf swing motion planning (optimal active torques) is calculated off line. Based on various performed simulation test, the GA's parameters used in this particular study are described as follows

a) Chromosomes representation

The Fourier series $\left\{a_{i}, a_{i}^{\prime}\right\},(i=1, n e)$, corresponding to active torques, represent the chromosome which is given by

$$
p=\left[a_{1}, \ldots, a_{n e}, a_{1}^{\prime}, \ldots, a_{n e}^{\prime}\right]^{\top} .
$$

The chromosomes are encoded to the continuous GA because it is inherently faster than the binary GA (the chromosomes do not have to be decoded prior to the evaluation of the cost function). This codification does not occupy a large memory space, knowing that the optimized 

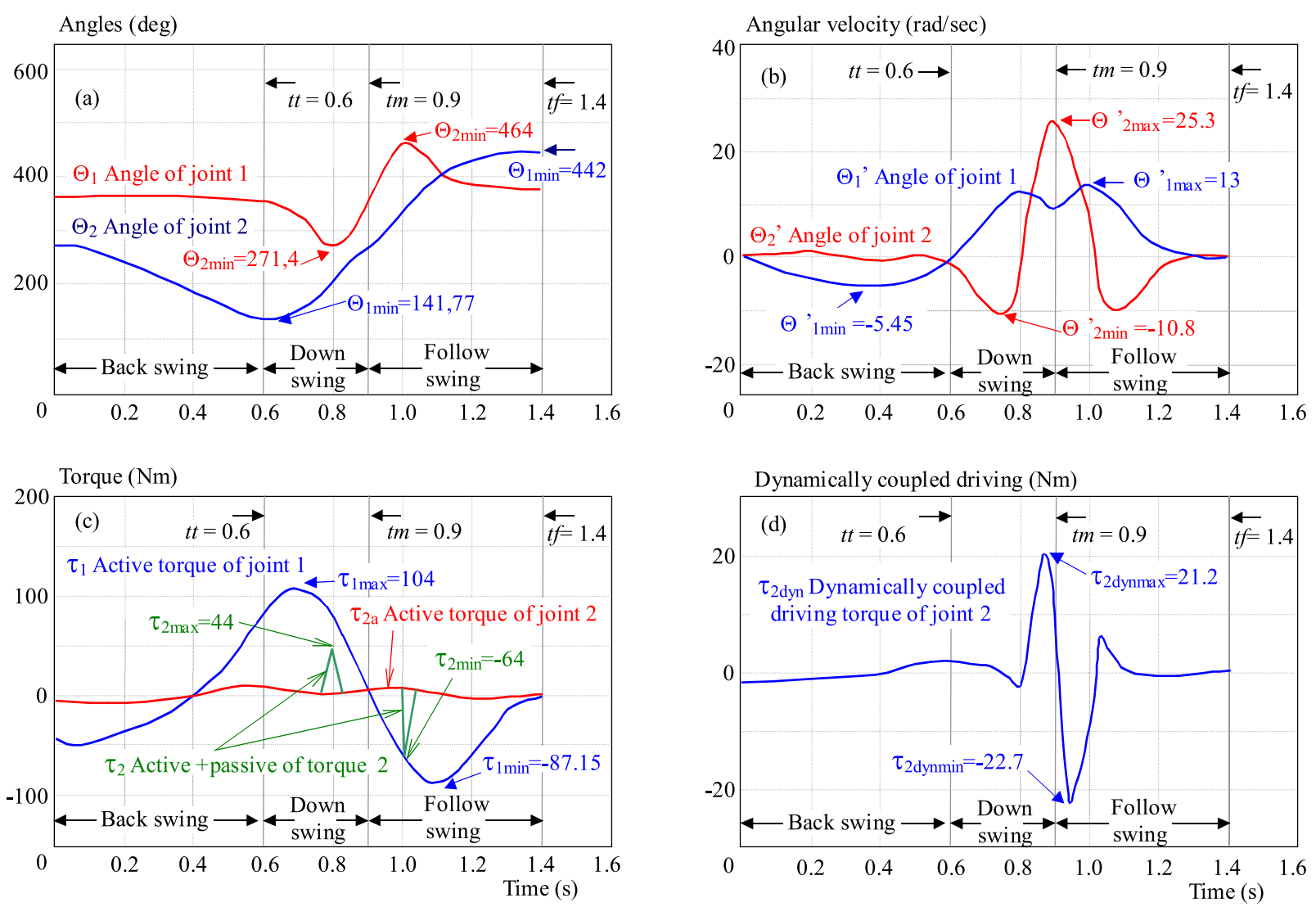

Fig. 3. Optimal motion planning by using GA with minimizing robot's energy consummation: (a) - angle, (b) - angular velocity, (c)torque, (d) - dynamically coupled driving torque of joint 2

variables are numbers with four digits after the decimal point.

b) Creating the initial population

Initially, the chromosomes are generated randomly.

c) Population size

We opted for a large population (200) because it allows GA to converge to the global optimum. However, if this population is chosen too small, it may be converging to a local minimum.

d) Generation size (mg)

As mentioned previously, the optimal motion of the robot is generated offline. Therefore the time-consuming issue do not cause any problem. For this reason, and in order to visualize the convergence of the optimal solution, we choose $m g=1000$, which is more than sufficient to obtain a near optimal solution. Usually, the final population will have a number of similar chromosomes that add validity to the optimal region and give more confidence in the final result [10]

e) Genetic Algorithm operations (selection, crossover and mutation)

The process of GA is accomplished by using roulette wheel selection which is the more used in the optimization problem [10]. In the literature, the most used values of mutation and crossover probabilities are respectively $\left(0.1 \%<P_{m}<5 \%\right)$ and $\left(6 \%<P_{c}<95 \%\right)[10]$. To avoid a random search, it is preferable to combine a medium value of the mutation probability $P_{m}=0.01$ with a high value of the crossover probability $P_{c}=0.9$, in order that the population will undergo a most important change. Both of these values are considered in this study.

f) Convergence check

The entire process is repeated until a maximum number of generations $(\mathrm{mg})$ is attained and consequently the GA should have reached the optimum. Based on performed simulation test, this number, and all other GA's parameters are assumed satisfactory.

As example, some optimal motion trajectories are shown and discussed bellow.

\subsection{Minimizing total consumed work and satisfy the boundary conditions}

The optimal values of Fourier series, $\left\{a_{i}, a_{i}^{\prime}\right\},(i=$ $1, n e)$, corresponding to active torques and impact speed of $35 \mathrm{~m} / \mathrm{s}$ are given by

$$
\begin{gathered}
a_{i}=\{-42.6501,-36.1723,-1.9223,80.5132,78.4099 \\
-58.9866,-62.9701\} \\
a_{i}^{\prime}=\{-5,8271,-5.5958,-1.8101,6.7533,0.6209 \\
5.8075,-3.7759,0\}
\end{gathered}
$$

The simulation results of optimal motion (torques, angle and velocity trajectories, dynamically coupled driv- 

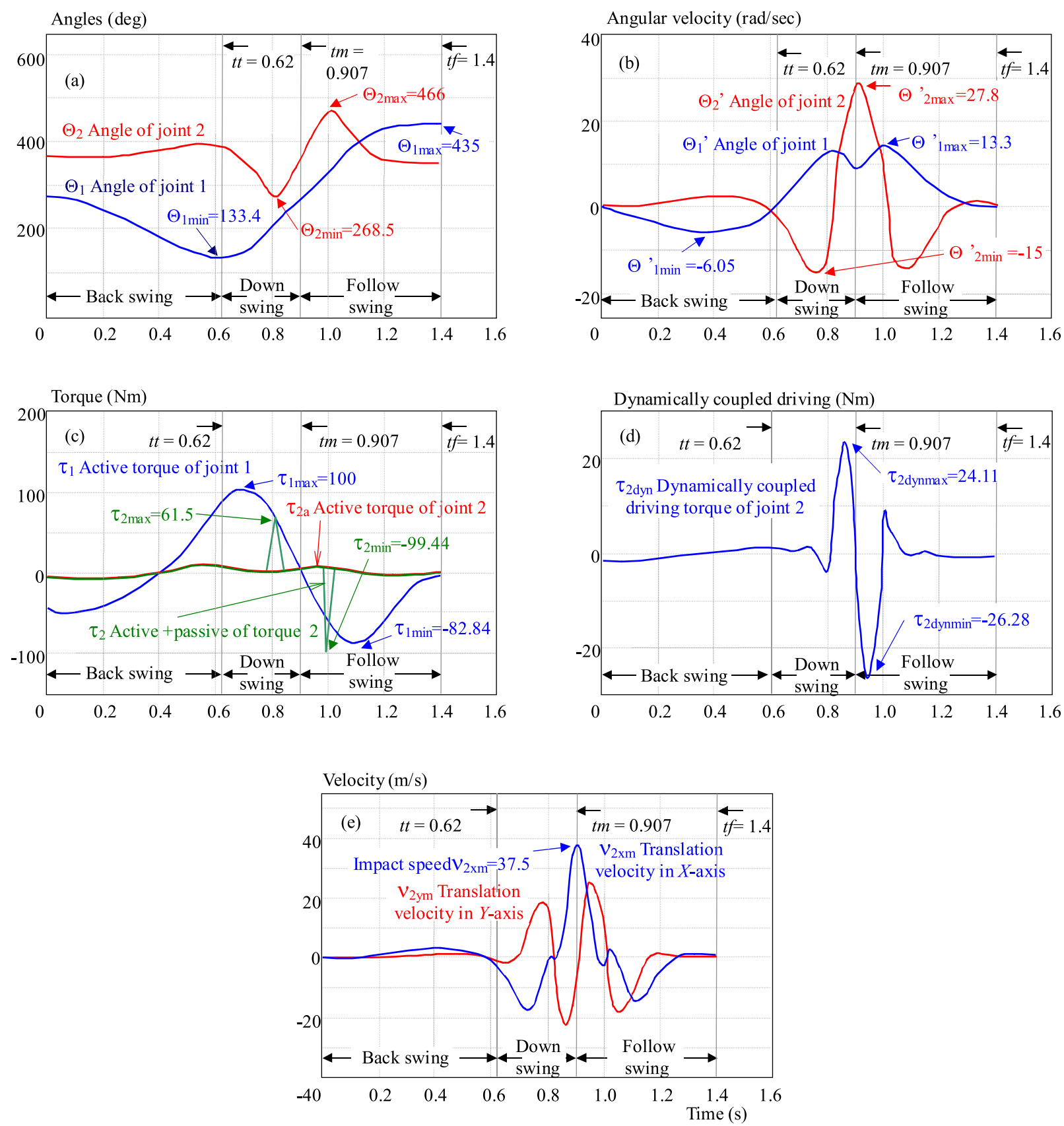

Fig. 4. Optimal motion planning by using GA with maximizing impact speed: (a) - angle, (b) - angular velocity, (c) - torque, (d) dynamically coupled driving torque of joint $2,-(\mathrm{e})$ - impact speed

ing) corresponding to the considered robot are shown by Fig. 3.

Figure 3(a) shows that the evolution of angular positions of both shoulder joint and wrist joint respect the boundary conditions and satisfy the kinematic conditions given by Eqs. (1) and (2). Figure 3(c) shows that active torques of joint 1 and joint 2 respect the physical conditions of motors. We can note also, that in downswing phase the joint stop generates the passive torque to accelerate the club in order to achieve higher swing speed. Contrary, in the follow-through phase the passive torque help the wrist joint to decelerate for stopping the club in final position.
In Fig. 3(b), we can see that at the beginning of the downswing phase, the shoulder joint accelerates first and the wrist joint accelerates later. However, just before the impact position, the shoulder joint decelerates to a local minimum speed and the wrist joint keeps accelerating rapidly until it reaches its (desired) maximum speed near the impact position. This multi-step acceleration phenomenon is due to the transfer of dynamically coupled driving from shoulder joint to a wrist joint. This second phase, downswing, is the more important period because it reflects the hyper dynamic motion of robot. In the following swing phase both of the joints decelerate and stop at the final time. 
Figure 3(d) shows the evolution of dynamically coupled driving and its influence on the rotation of the wrist joint.

\subsection{Maximizing impact speed and satisfy the boundary conditions}

The robot is characterized by it hyper dynamic manipulation with high speed. For that reason, the impact speed of golf swing was maximized in optimal motion generation subject to constraints in rotation range and active torque.

The optimal values of Fourier series, $\left\{a_{i}, a_{i}^{\prime}\right\}, \quad(i=$ $1, n e)$, corresponding to active torques and maximum impact speed are given by

$$
\begin{gathered}
a_{i}=\{-39.6078,-39.6826,-0.5215,79.2278, \\
77.3180,-56.3304,-59.3124,0\} ; \\
a_{i}^{\prime}=\{-6.0173,-5.5518,-0.1802,4.1250,0.9643, \\
4.0520,-0.5011,0\} .
\end{gathered}
$$

The simulation results of optimal motion (torques, angle and velocity trajectories, dynamically coupled driving, translation velocity) corresponding to the considered robot are shown by Fig. 4 .

Figure 4(a) shows that the backswing phase of the swing motion is larger than the one shown by Fig. 3(a) and the shoulder joint reaches it minimum angular position. Figure 4(c) shows that the active torque of the shoulder joint reaches 100 N.m and remains close to its upper limit for a long period during the downswing phase, thus maximizing the impact speed. We can see also the passive torque, generated by the joint stops in the downswing and the follow swing phases when the wrist joint reaches its limits (joint stops). In Fig. 4(b) the multi-step acceleration is again observed and Fig. 4(d) shows that the dynamically coupled driving torque becomes greater in the downswing phase to help the club to achieve the impact position with high speed. In Fig. 4(e), we can see the maximum impact speed $\left(v_{2 x m}=37.5(\mathrm{~m} / \mathrm{s})\right.$ obtained with our method.

\section{CONCLUSION}

In this study, optimal motion planning issue for a golf swing robot is investigated. The manipulator is characterized by its anthropomorphism mechanism based on an ingenious structure which is inspired from the professional golfer's arm. A new method based on Fourier series approximation and Genetic Algorithm is proposed to generate the optimal high speed motion of robot. The problem is solved as a multi-objective constrained optimization with boundary conditions to satisfy and objective function to minimize.
Simulation results show the effectiveness of Genetic Algorithm method to solve the optimal motion planning issue.

\section{REFERENCES}

[1] XU, C.-Ming, A.-NAGAOKA, T.-ShimojO, M. : Motion Control of a Golf Swing Robot, In Journal of Robotic and System, vol. 56, pp. 277-299 February (2009).

[2] XU, C.-MING, A.-MARUYAMA, T.-ShimoJO, M. : Motion Generation for Hyper Dynamic Manipulation, Journal of Robotics and Mecatronics 17 (May 2007), 405-416.

[3] SUZIKI, S.-INOOKA, H.: Golf Swing Robot Emulating a Human Motion, In Proceedings IEEE International Workshop of Robot and Human Communication, 1997, pp. 28-33.

[4] SUZIKI, S.-INOOKA, H. : A New Golf Swing Robot Model Emulating Golfer's Skill, Sports Engineering 2 (1999), 13-22.

[5] HOSHINO, Y.-KOBAYASHI, Y.-YAMADA, G. : Vibration Control using a State Observer that Considers Disturbances of a Golf Swing Robot, International Journal of JSME (2005), 48-60.

[6] MING, A..-KAJITANI, M.: Human Skill and Ultra High Speed Manipulator, In Proceeding of the $3^{\text {rd }}$ France-Japan Congress and $1^{\text {st }}$ Europe-Asia Congress on Mechatronics, 1996, pp. 436-441.

[7] MiNG, A.-KAJITANI, M.: Human Dynamic Skill in High Speed Actions and its Realization by Robot, Journal of Robotics and Mechatronics (2000), 318-334.

[8] MiNG, A.-MITA, T.-DHLAMINI, S.-KAJITANI, M. : Motion Control Skill in Human Hyper Dynamic Manipulation - an Investigation on the Golf Swing Simulation, IEEE transaction of Robotics and Automation (Aug 2001), 47-52.

[9] MING, A.-HARADA, N.-SHIMOJO, M.-KAJITANI, M. : Development of a Hyper Dynamic Manipulator Utilizing Joint Stops, Proceedings IEEE/RS of Intelligent Robots and Systems (Oct 2003), 2084-2089.

10] SIVANANDAM, S. N.-DEEPA, S. N.: Introduction to Genetic Algorithms, Springer-Verlag, Berlin Heidelberg, 2008.

Received 29 April 2011

Souhila Aboura received her MS degree in Automatic Control from the University of Sciences and Technology of Oran, Algeria, in 2009. She is currently PhD candidate in Electrical Engineering at the same university. Her research interests include Automatic control, optimal motion generation, as well as Optimization.

Abdelhafid Omari received his $\mathrm{PhD}$ in Electrical Engineering from the University of Electro-Communications in the city of Tokyo, Japan in 2001. He is currently working as an Associate Professor in the Faculty of Electrical Engineering at the University of Sciences and Technology in the city of Oran in Algeria. His research and teaching interests include: Automatic control, Intelligent Mechatronic Systems and Robotics control.

Kadda Zemalache Meguenni received his $\mathrm{PhD}$ in Electrical Engineering from the University of Evry val d'Essonne, France in 2006. He is currently working as an Associate Professor in the Faculty of Electrical Engineering at the University of Sciences and Technology in the city of Oran in Algeria. His research and teaching interests include: Intelligent control, Mechatronics and robotics control. 